$3 D$ PRINTING OF PERSONAL

PROTECTIVE

EQUIPMENT AND

REPURPOSING OF

COMMON

HOUSEHOLD AIR

FILTERS

VERSION 1

MAR 26, 2020

open Access

DOI:

dx.doi.org/10.17504/protocol s.io.bd77i9rn

Protocol Citation: Giana Schena, Emma Murray 2020. 3D Printing Of Personal Protective Equipment And Repurposing Of Common Household Air Filters. protocols.io https://dx.doi.org/10.17504/p rotocols.io.bd77i9rn

License: This is an open access protocol distributed under the terms of the Creative Commons Attribution License, which permits unrestricted use, distribution, and reproduction in any medium, provided the original author and source are credited

\section{(3) 3D Printing Of Personal Protective Equipment And Repurposing Of Common Household Air Filters V.1}

In 1 collection

Giana Schena ${ }^{1}$, Emma Murray ${ }^{1}$

${ }^{1}$ Temple University

Coronavirus Method Development Community

Giana Schena 
Protocol status: Working

Our group has created working models for the PPE designs in this protocol. We are currently coordinating with private businesses in the Philadelphia region to optimize and mass produce the equipment in this protocol to aid in the protection of hospitals workers on the front line. We are constantly optimizing and updating our models and protocols to ensure both the creation of the most protective PPE and implementation of efficient methods of delivery. New versions will be shared with the community as signif

Created: Mar 25, 2020

Last Modified: Mar 26, 2020

\section{PROTOCOL integer ID:} 34783

Keywords: PPE, Personal protective equipment, Covid19, Coronavirus, Face mask, 3D, Printing

\section{ABSTRACT}

During the height of the COVID-19 outbreak, personal protective equipment shortages are felt around the world, in spite of the fact that traditional face masks are not even sufficient to fully protect against the 0.125 micron SARS-CoV-2 virus. Medical personnel, more than any other sector, are being hit hardest by this shortage, given their close contact with infected persons. During this time of crisis and shortage, alternative and atypical solutions are necessary to ensure the protection of those who are on the front lines against this disease. One solution is the creation of reusable 3D-printed personal protective equipment (PPE) in conjunction with appropriate disposable filtration.

3D-printed face masks are available on a variety of different open source 3D-printing online platforms. Unfortunately, the general public remains largely unaware of these potential solutions and can also be intimidated by the lack of information available regarding how to integrate scientifically appropriate filters. The intention of this white paper is to make available the information needed for simple construction of effective 3D-printed masks. We recommend a 3D printed face mask design made available by CREALITY, a leader in 3D-printing machines. By using CREALITY's open source designs and easily obtainable air filters, cut to a desired size, we can increase our ability to protect medical workers against this disease. Additionally, CREALITY offers designs for a 3D-printed eye mask that can be used with plexiglass to protect eyes against airborne droplets. In practice, CREALITY's 3D model object STL files can be uploaded into any available slicer software allowing for creation of specific commands for 3D printers. Within the slicer, face mask and eye mask models can be scaled to small, medium, or large to fit a variety of face sizes. We have also included strap adjustment clip models for use with facemasks and a face shield to extend the life of traditional facemasks.

We also propose a plan to coordinate large-scale construction of crowd-sourced 3D printed masks with collection and delivery of said masks to at-risk businesses, in particular hospitals and care facilities.

\section{ATTACHMENTS \\ COVID19_PLAN_FOR_3D_ \\ PRINTING_OF_PERSONA \\ L_PROTECTIVE_EQUIPME \\ NT_AND_REPURPOSING_ \\ OF_COMMON_HOUSEHO \\ LD_AIR_FILTERS.pdf}

\section{GUIDELINES}

\section{CREATION OF 3D PRINTED PERSONAL PROTECTIVE EQUIPMENT}

In order to streamline access to acceptable 3D printed models, all models found online have been uploaded into the following Google Drive (STL_files.zip). The writers of this plan did not design these models nor intend to take away credit from 
the original makers for the time and skill that went into making these models. Credit goes to Creality, $\mathrm{HanochH}$ at Thingiverse and Tadziurski at Thingiverse.

The WHO and $\underline{\mathrm{CDC}}$ advise the use of $\mathrm{N} 95$ surgical masks or respirators as well as eye coverage for protection against aerosolized particles or airborne droplets during surgical procedures, while the $\mathrm{WHO}$ advises using a face mask for non-aerosol inducing patient care. Face shields have been proposed as a way to extend the life of face masks and surgical masks as efficacy of these products drops when exposed to moisture.

To extend the use of N95 surgical masks, full N95 face masks can be cut in half to fit into the 3D printed units. There are some high quality HEPA filters which are designed to filter out particles smaller than 0.1 microns and may also be applicable for repurposing if the $\mathrm{n} 95$ shortage becomes dire.

Additionally, creation of a general facemask, not an N95 surgical mask, can be done via 2 methods: either by repurposing general home air filters such as HEPA filters, which filter down to 0.3 microns, or by cutting medical facemasks into smaller segments which fit into the 3D printed designs. While the SARS-CoV-2 virus may be smaller than the repurposed filtration, these filters will still provide a more effective physical barrier than those provided by general facemasks. Additionally, with the use of adhesive foam strips and elastic bands the mask will have a more secure fit than general facemasks, increasing protection.

Eye protection and face shields can be created using 3D printed framework, plexiglass or acrylic sheets, adhesive foam strips, and elastic banding.

Designs are proposed below.

\section{FACE MASK CREATION}

Face masks can be printed using a variety of different 3D models. Here we highlight two different versions which allow for the removal of disposable air filtration systems such as medical face masks or commercial air filters. In comparison to general face masks and N95 grade surgical facemasks, the 3D-printed face-covering has more plastic surface area than general surgical masks and still allows for easy breathing, due to the shape of the respirator openings. This is beneficial because it allows for less air filtration products to be used, i.e. a general face mask can be cut into 4 sections and used in 4 different facemasks, greatly extending the use of each hospital's current stock. After printing, adhesive foam weather stripping can be added to the perimeter area which is in contact with the medical personnel's face to create a more comfortable snug fit. Additionally, each model contains slots for inclusion of maximum 1/2" elastic banding for tightening on the face.

\section{REUSING EYE- AND FACE-MASK DESIGNS}


Once an individual is finished using the 3D printed objects on their shift, objects can be soaked in bleach to remove any possible contaminants before reuse. Adhesive stripping, elastic bands, and filters can be properly disposed of prior to cleaning. Once clean, new disposable materials can be added. Additionally, if enough masks are printed, each person can keep the mask and replace the filters at regular intervals.

\section{COST}

Using generic PLA, face masks and eye masks will cost $\sim \$ 1$ each to print. Print time can vary depending on the machine. On a home machine, each print can take 5-6 hours. Printing completion time may be decreased on larger scale 3D printers. While this process may not be as expedient as desired, by rapidly initiating production and using as many crowd-sourced 3D-printing facilities as possible, creating enough PPE in this time of need is certainly possible. Adhesive foam strips and elastic bands are inexpensive on sites such as amazon or hardware and craft websites and allowing $1 \mathrm{ft}$ of each product per "use" gives a cost of $\sim \$ 0.50$. Plexiglass sheets for eye masks can survive multiple uses with bleaching. This makes the most cost-intensive production step the purchase of adequate air filtration products, which can vary depending on the desired micron filtration levels from low to high viral filtration.

\section{ENLISTING THE PUBLIC}

Increasing the supply of eye and face masks for hospital use can be accomplished through enlisting the help of public companies, universities, schools, and 3D printing hobbyists to fulfill the demand. To do so, emails can be sent to companies with a request for $3 \mathrm{D}$ printed materials or consumables depending on the company's forte. Enlisting the public, universities, and schools provides a different host of issues. During this time, we are significantly reducing viral spread by decreasing public interaction. Given this logistical complication, we suggest public drop-off locations for home-printed masks should be made available at locations in which limited public outings are permitted, such as grocery stores.

We suggest enlisting the aid of either law enforcement or hospital workers who drive by these locations on their way to work to facilitate the delivery of 3D printed materials to in-need locations.

Before use, all materials should be sterilized to ensure they are free of any virus particles which they may have been exposed to during either production or transportation.

\section{BEFORE START INSTRUCTIONS}

Assembly should be performed wearing gloves and a face mask.

\section{Two Piece Face Mask Printing and Assembly}


1 Open the facemask_face_unit.st/file (size= currently female size, would need to be scaled up).

a. Ensure that support is on

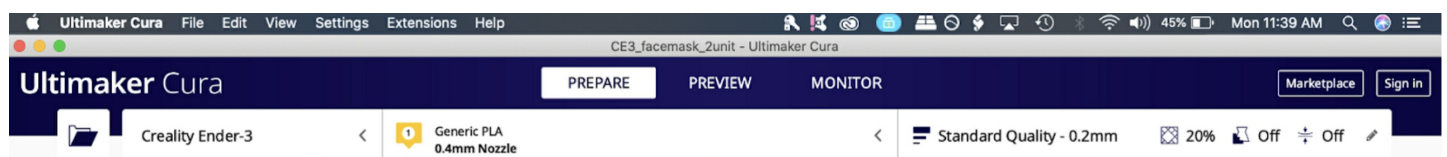

\section{Two Piece Face Mask}

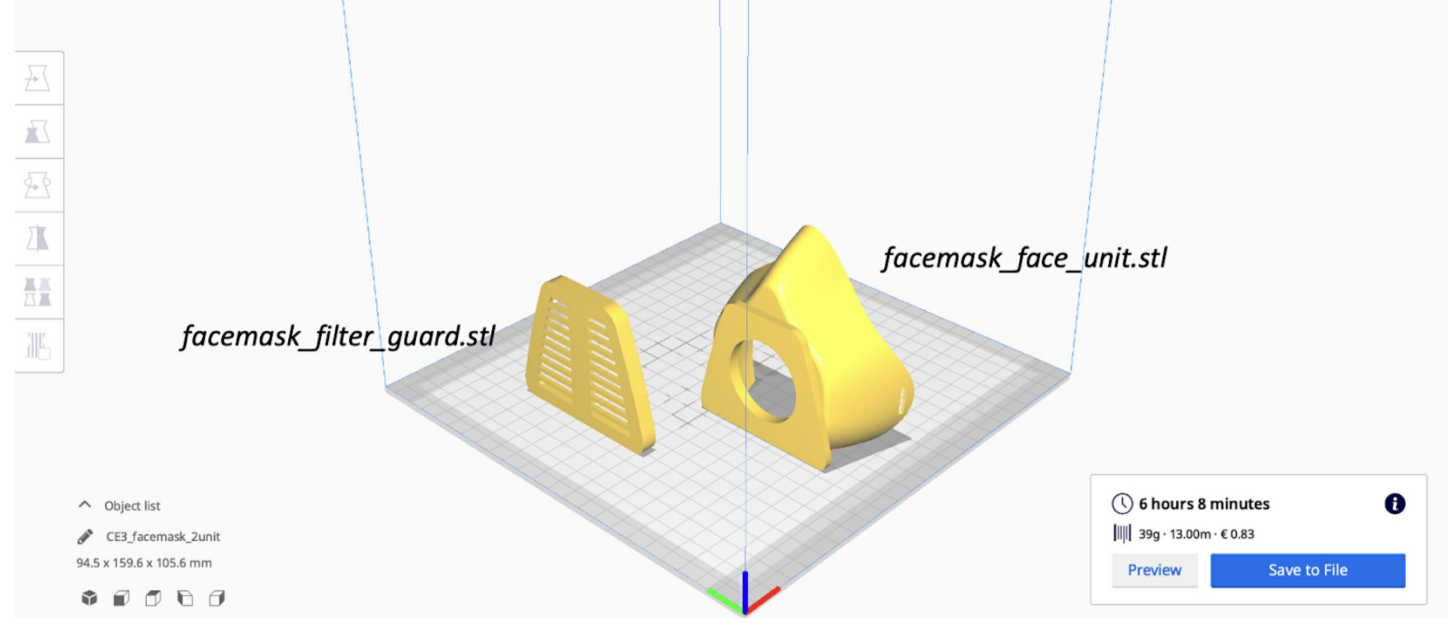

2 Open the facemask_filter_guard.st/file (currently on scale with the face unit, would also need to be scaled up).

a. Support is not necessary

b. Guard is in shape of a trapezoid: the face of the guard is 2.5 inches tall with 2.75 inch base length and 1.6 inch top length

3 Open facemask_clip.st/file and print two copies.

Note

Set $\mathrm{XYZ}$ percentages to $54 \%$.

4 Ensure the following settings are on in your printer (Exact wordage for Ultimaker Cura but similar settings available on all slicers):

a. Infill density $=30 \%$

b. Generate Support $=$ Checked

i. Support Placement $=$ Touching Buildplate

ii. Support Overhang Angle $=45$ degrees

$5 \quad$ Slice file and print. 
6 Soak the unit and guard in Clorox bleach for 00:05:00. Rinse well and air dry.

7 Choose filter material:

a. For general face mask replacement: option 1

b. For higher levels of protection: option 1 or option 2

c. Peel filter off of metal webbing, discard metal webbing and keep filter material: 


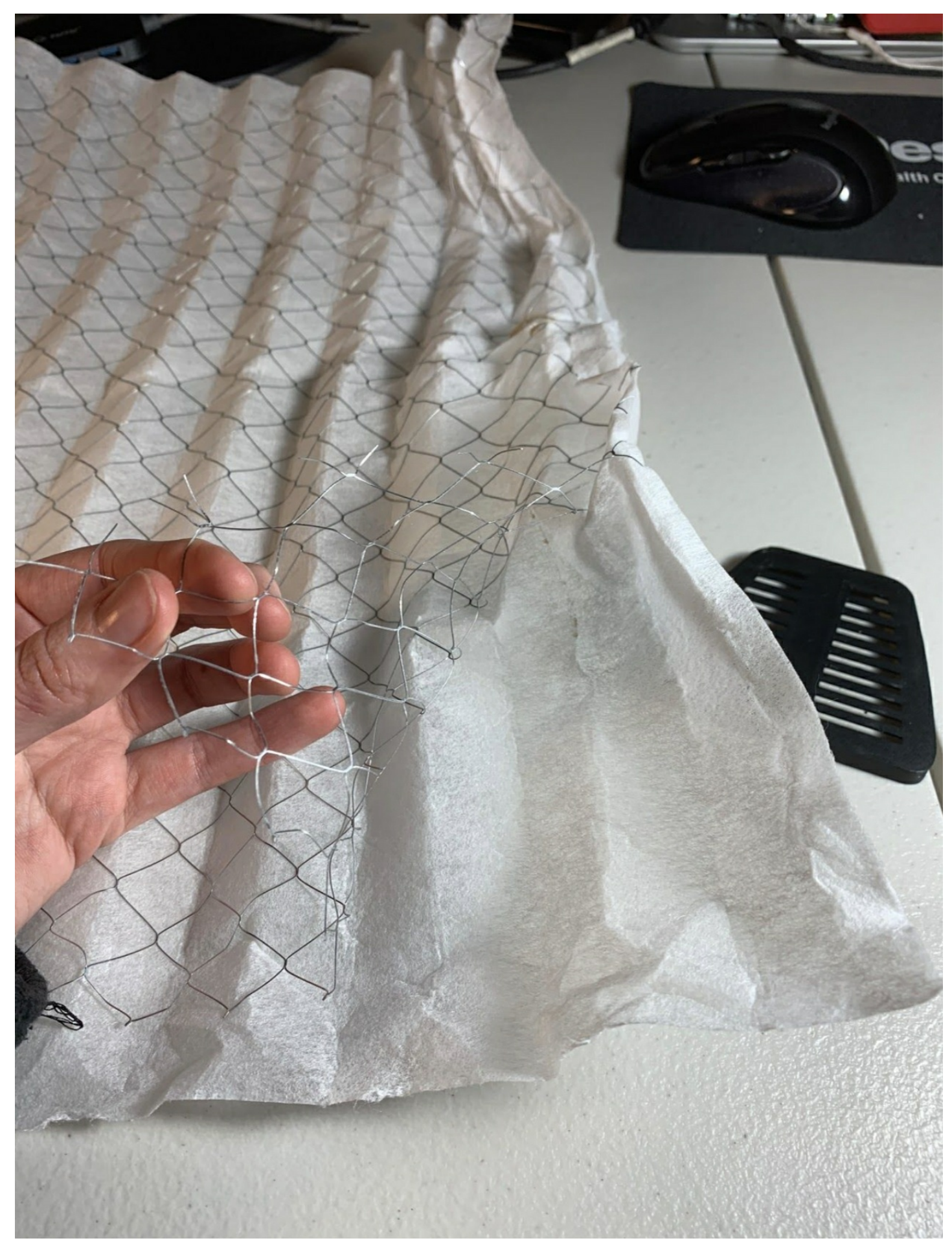

8 Cut filter to fit within the guard: 


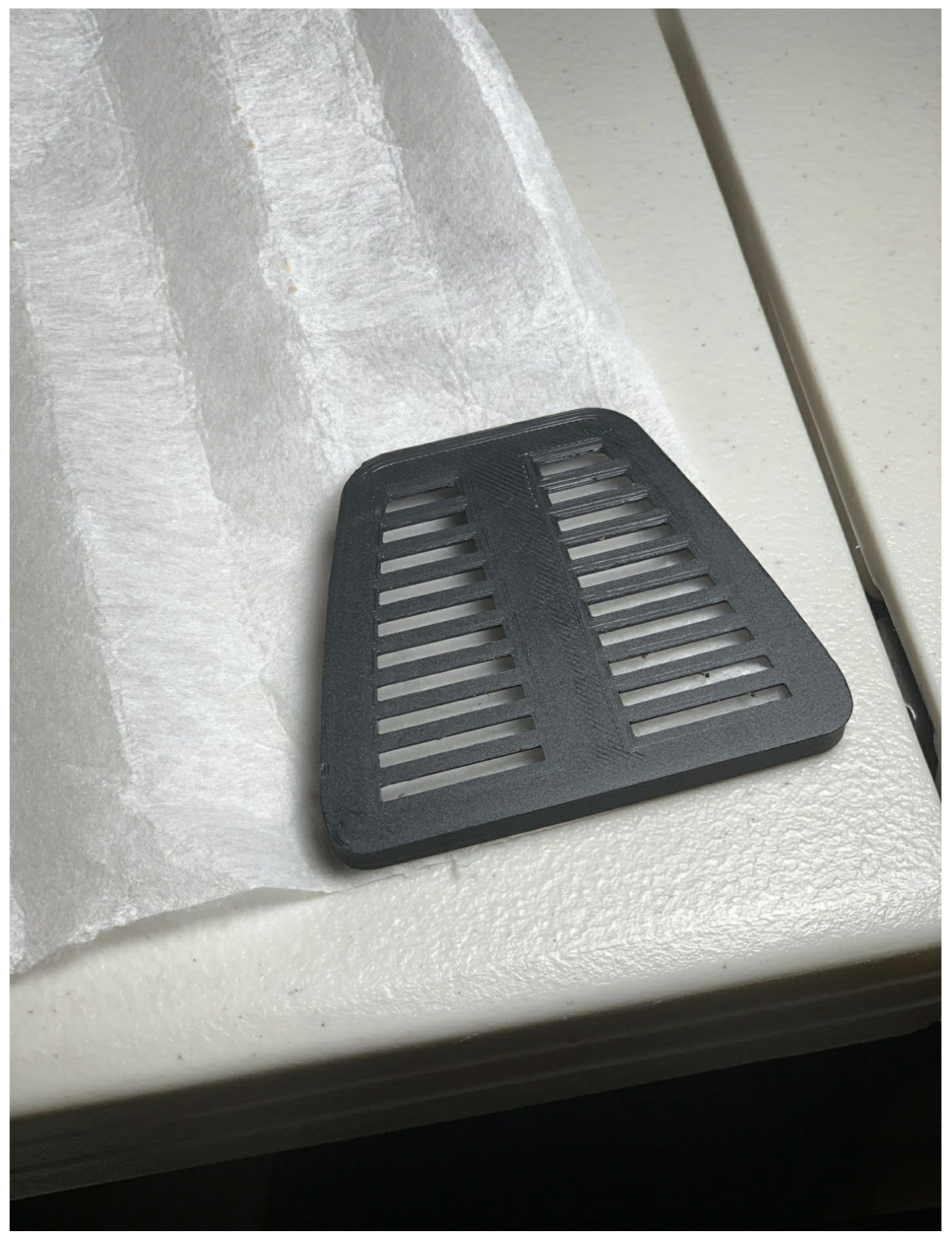

9 Place filter between the guard and the face unit and snap together.

Note

The facemask_filter_guard.st/fits very tightly on the facemask_face_unit.st/but can still be removed for reuse by pressing against the guard from inside the face unit. 

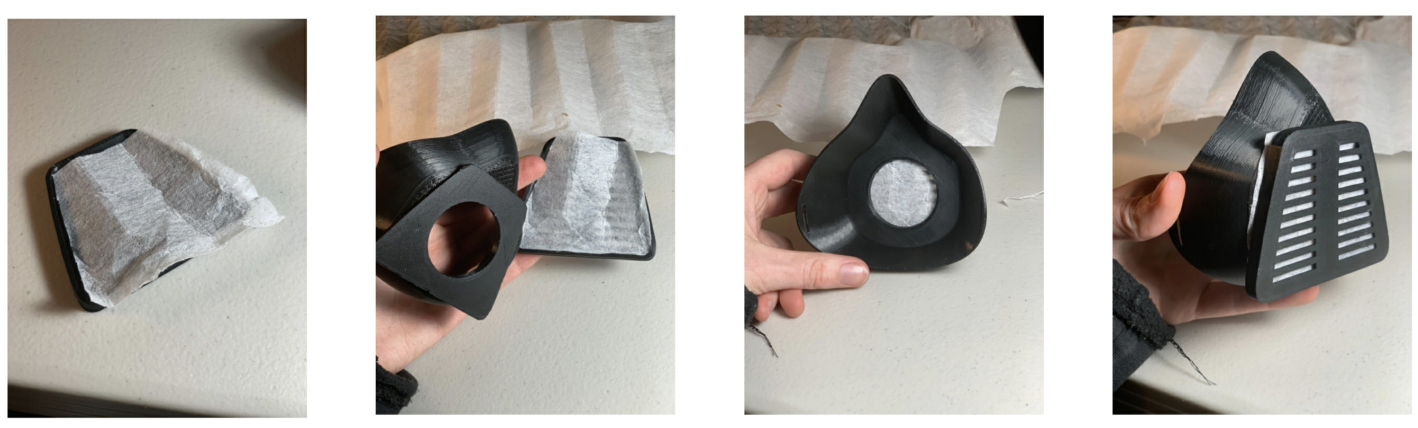

10 Thread elastic band through clips: 


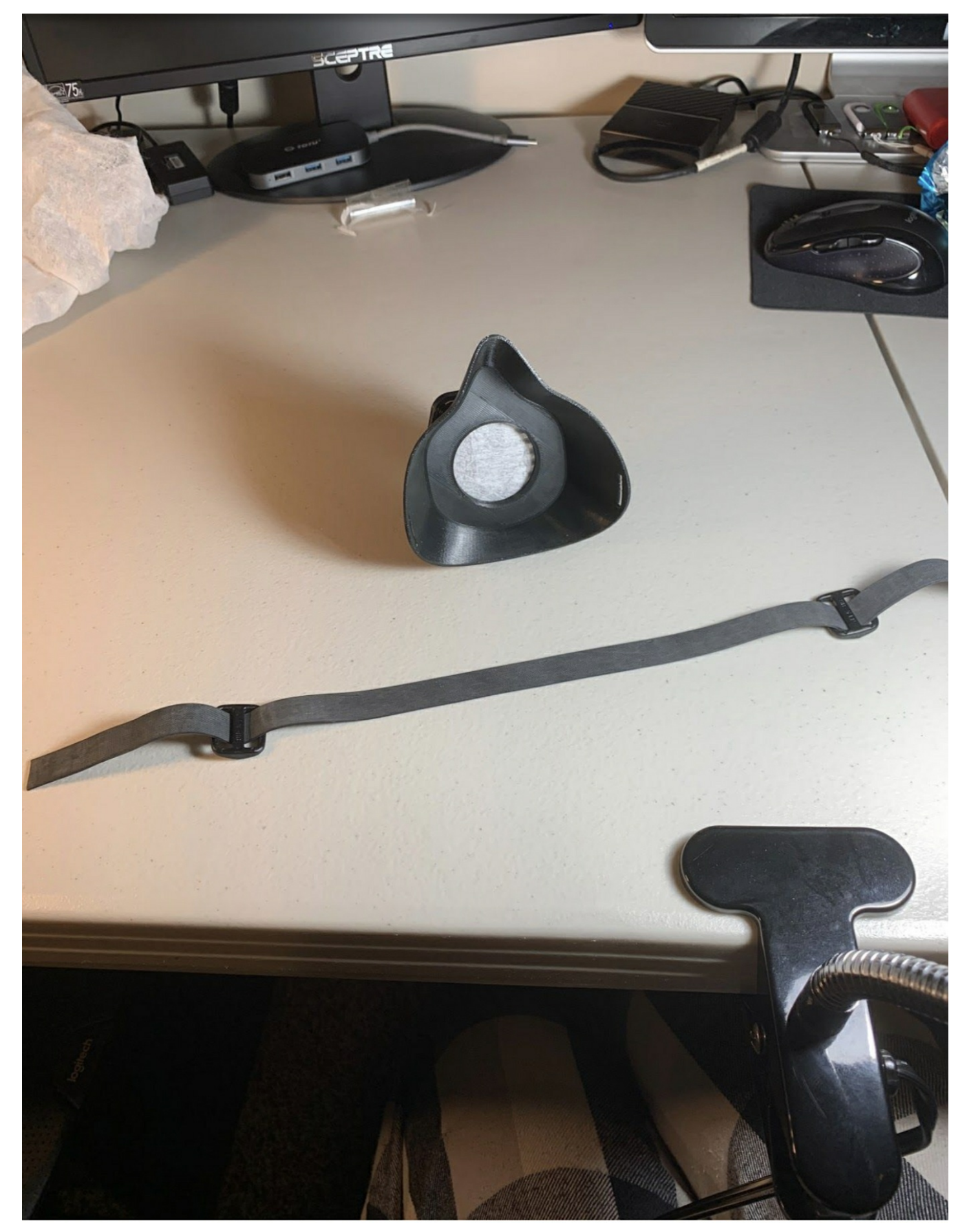

11 Attach elastic band to holes on either side of the face unit: 

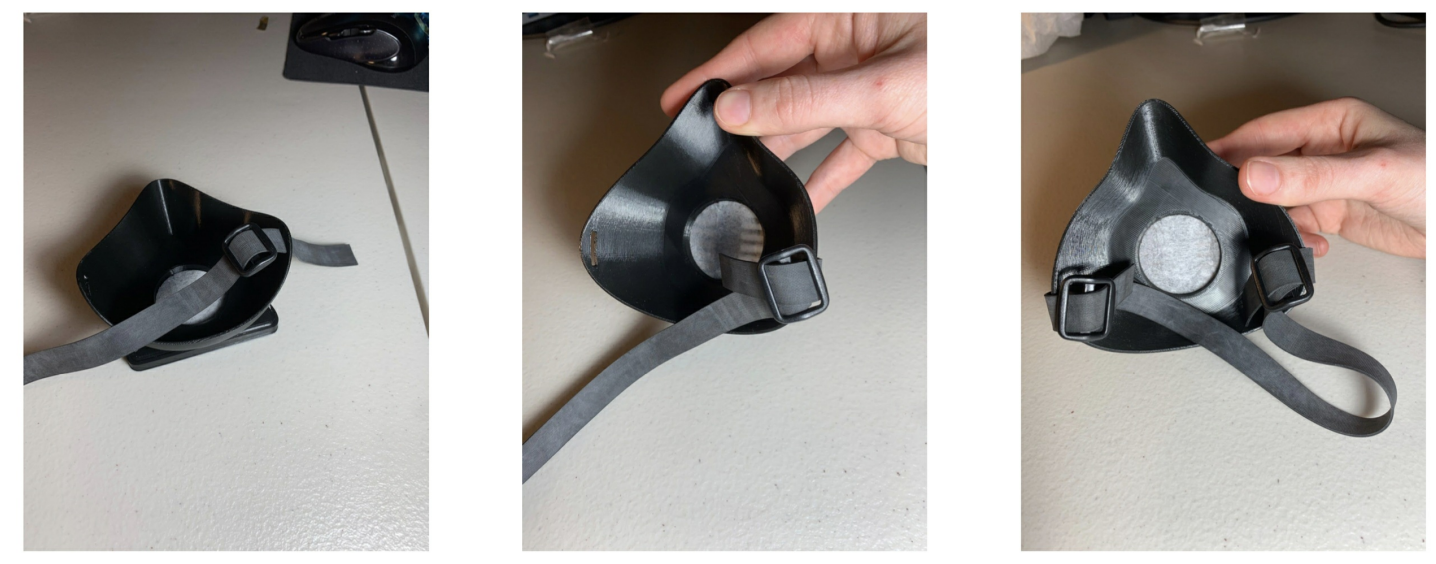

12 If desired, padding can be taped or glued around the outside edges of the facemask.

\section{One Piece Face Mask Printing}

13 Print the facemask_single_unit.st/file.

Note

File should be printed with support enabled. 


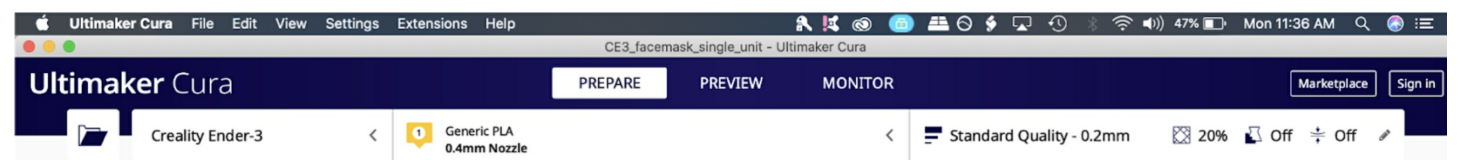

\section{Single Piece Face Mask}

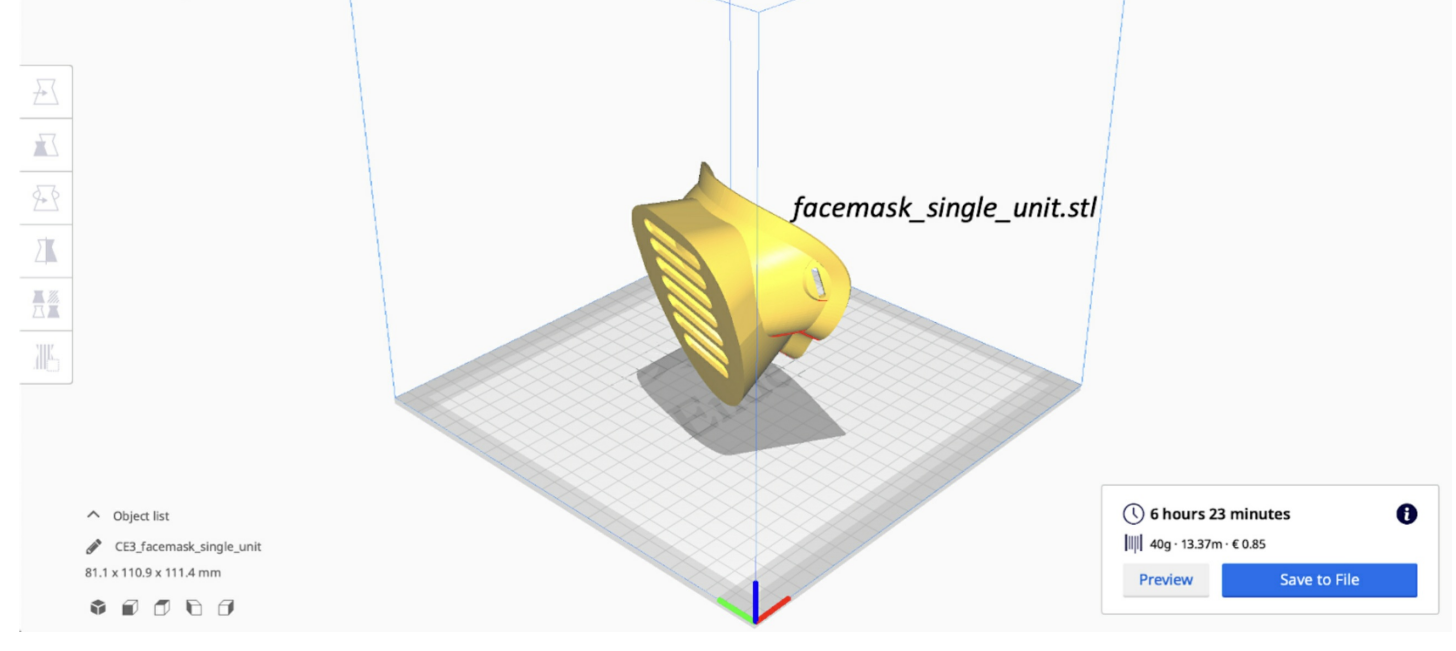

14 Print two clips using the facemask_clip.st/file with XYZ percentages set to $54 \%$.

15 Cut air filtration material to fit inside the single unit.

16 Adhesive foam weather stripping and elastic banding can then be added to create a snug fit on the face.

17 Two clips can be printed using the facemask_clip.stl file with XYZ percentages set to $54 \%$.

18 Air filtration material should be cut to fit inside the single unit. 
19 Adhesive foam weather stripping and elastic banding can then be added to create a snug fit on the face.

\section{Three Piece Face Goggles Printing}

20 Print eye goggles using the eyemask_thick.stl, eyemask_thin.st/and eyemask_hook.st/files.

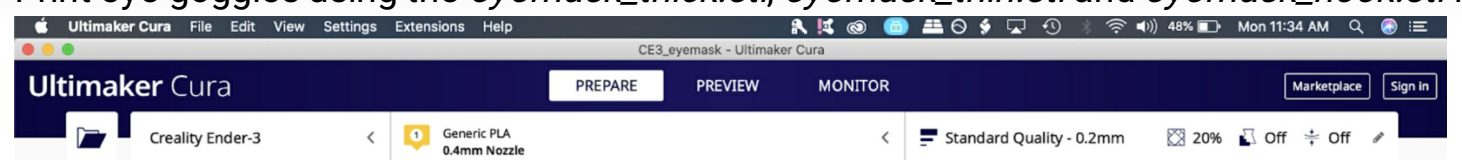

\section{Three Piece Face Goggles}

Object list

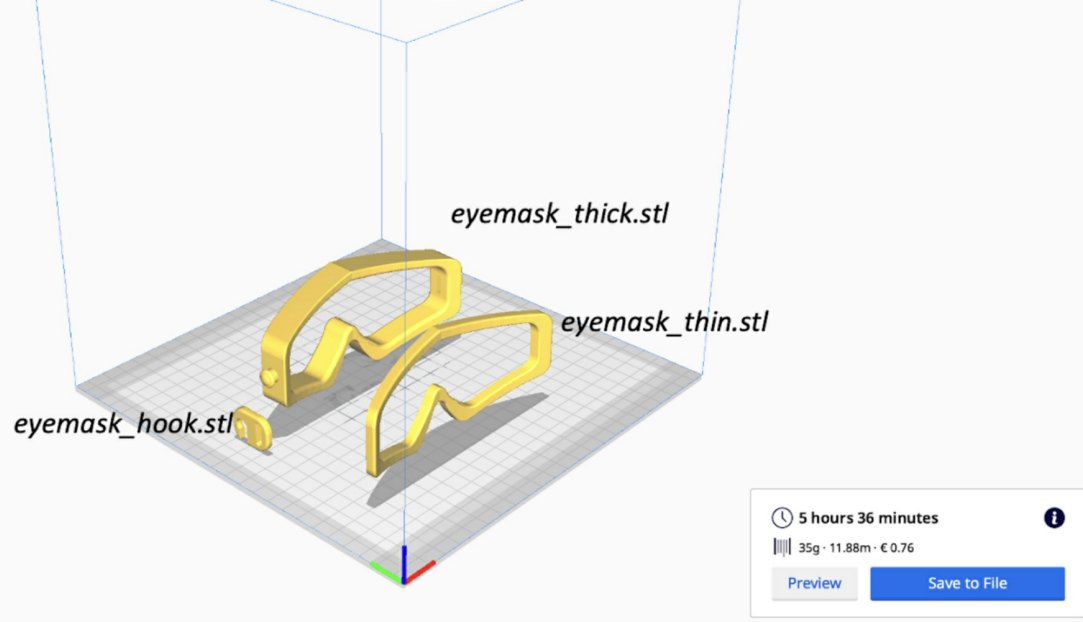

21 Cut and glue plexiglass or A4 acrylic sheets in between the eyemask_thick.st/(outer piece) and eyemask_thin.st/(inner piece) prints.

22 Foam-adhesive weather stripping can be attached to the inside of the eyemask_thin.st/print for a more comfortable and secure fit on the face.

23 Clip the eyemask_hook.st/print to the eyemask_thick.st/print and attach maximum width of 1/2" elastic banding for strapping the mask to the face. 


\section{One Piece Face Shield, No Screws Printing}

24 Create a face shield using the faceshield_one_unit.st/file, originally found on Thingiverse and made by $\underline{\mathrm{Hanoch}}$.

Note

This face shield is ideal because no screws or clamps are required. The model's design uses tension to keep any acrylic sheets firmly in place.

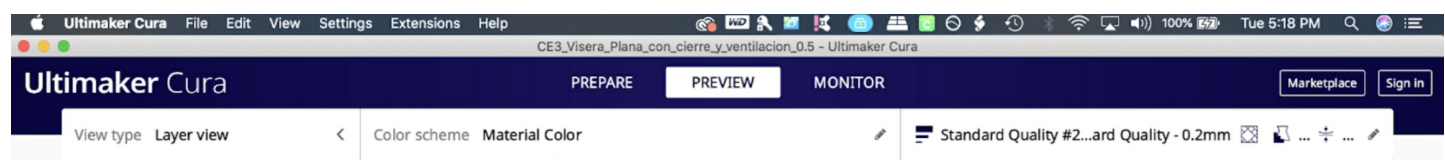

\section{One Piece Face Shield}

CE3_Visera_Plana_con_cierre_y_ventilacion_. 0.5

$145.6 \times 164.8 \times 15.0 \mathrm{~mm}$

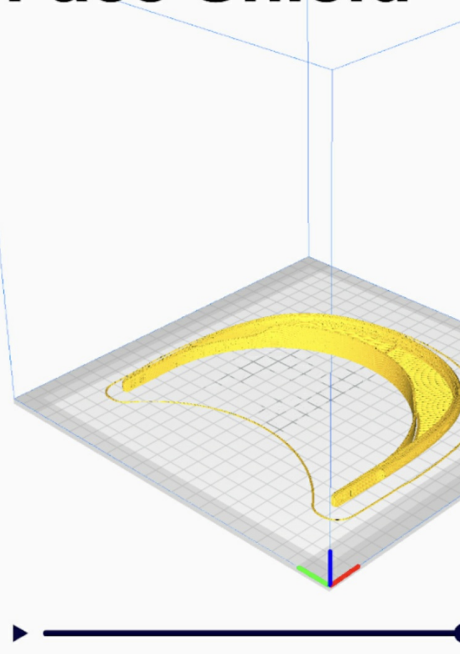

faceshield_one_unit.stl

(1) 3 hours 13 minutes

|li|| $22 \mathrm{~g} \cdot 7.26 \mathrm{~m} \cdot 60.46$

Save to File

25 Cut a sheet of acrylic to run along the diameter of the opening.

Note

This design is made to fit $A 4$ sheets and the original makers tested to confirm this design works with $0.10 \mathrm{~mm}$ and $0.24 \mathrm{~mm}$ thickness sheets. A video of the mask in use can be seen on the makers thingiverse. 